\title{
DESIGN AND IMPLEMENTATION SMART TRAFFIC LIGHT USING GSM AND IR
}

\author{
Thaar A.Kareem ${ }^{1}$

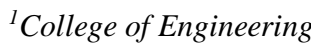 \\ University of Misan, \\ Misan, Iraq. \\ Thaar_kareem@uomisan.edu.iq
}

\author{
Mays Kareem Jabbar ${ }^{2}$ \\ ${ }^{2}$ College of Engineering \\ University of Misan, \\ Misan, Iraq. \\ m_mays85@uomisan.edu.iq
}

\begin{abstract}
The increasing in the number of vehicles on streets has led to traffic congestion. In order to reduce the waiting time in cases of emergency, the idea of this work is suggested. This work is divided into two parts, the particular part and software part. The first circular particular part is a model which consists of four lanes junction of a traffic light, it also has GSM system ( Global System for Mobile Communications). The GSM and lamps of the traffic light are connected to Arduino UNO. The Arduino controls every signal which is coming from the inputs (GSM) to software and display to the outputs (lamps) The second circular particular part is a model which consist same components the first circuit except replace the GSM with IR(infrared Remote).The goal from this work is to help us in the emergence cases, the opening and closing of the traffic light are controlled by using GSM system and IR, the time of each lane, is controlled that means reduce the crowding.
\end{abstract}

\section{KEYWORDS: TRAFFIC LIGHT, ARDUINO UNO, GSM, IR}

\section{INTRODUCTION}

During the time when pedestrians, buggies, and wagons were roaming the roads, the world's first traffic lights was installed in London in the 1868, long before the automobile was invented., It was consisted of a lantern, illuminated by gas and, with two signals of red and green which represented stop and caution, respectively. On January 2, 1869, this crude traffic light exploded, injuring the policeman who was operating it. Despite the primitively and the number of accidents, especially those such as the explosion of the traffic light on January 2nd, 1869, the situation was not as worse as the time when the automobiles era has begun. One of the earliest solution was to adapt railroad signals to be used in streets, that was first thought by a police officer from Detroit, Michigan (officer William L. Potts). However, major challenge has come out as the railroads run along parallel lines, while streets travel at right angles. The world's first 4-ways three color traffic light (red, amber, and green) connected throughout wires and electrical controls. In 1920 it was installed in a corner at Woodward and Michigan Venus in Detroit. A year later, the success of the experiment in the organization of vehicle traffic Fifteen lamps were installed in Detroit and all were automatic. At the same time, Morgan, a talented American inventor, realized the need to control traffic in Cleveland, Ohio, the inventor of electric light. Although at first they were similar to existing train stations [1].

The numbers of vehicles are increased because of the increasing in population numbers. This led to full congestions in streets and intersections. In some countries, for example Iraq, the streets inside the cities are narrow and there are no highways that led to increasing in traffic congestion which may reach up to 15 minutes at the intersection. And thus leading to delay in emergency like ambulances and fire trucks ...ect.

In [2] the authors made the time of traffic light unfixed according to the size of traffic jam, when there is a traffic jam in any road the green light which means permeation gives full time to the user of the road. If there is no traffic jam, the green light does not give full time. In [3] the authors used method to help reduce congestion on roads and to help in coping with accidents as the heavy vehicles and light vehicles will be in different lanes, this proposed system made roads a safer place to travel. In [4] the author solved the traffic congestion issue by developing a new traffic light control system controlled using programmable logic and then implements the system on a model of a traffic light controller (PLC).

in (11) traffic jams have been reduced using sensor technology, where the time of the signal is changed based on the number of vehicles on the road. The system calculates congestion in the road if the number of cars is many times 
the time of the green signal and gives it extra time until the congestion is eliminated, However, if the number of cars is small, it gives the system time

The goal of this work is to help us in the emergence cases and to reduce the crowding, where we can control in the opening and closing of the traffic light by using GSM system and IR, and also we can control the time of each lane.

The remainder of this paper is organized as follows: Section 2 presents the first circuit design using GSM, Section 3 presents the second circuit design using IR. The proposed system is presented in Section 4, Section 5 is a comparison between the two circuits, and the conclusion of this work is presented in Section 6.

\section{The First Circuit Design USING GSM}

Main aim of this work is to design a traffic light controller. For that purpose, a circuit was implemented which had 12 LEDs in a 4- way intersection. In each intersection there three LEDs each of yellow, green and red colors.

Each intersection further has 4 lanes. Current limiting resistors of $1 \mathrm{k} \Omega$ connect each LED to Arduino UNO's digital I/O pin according to the circuit diagram.

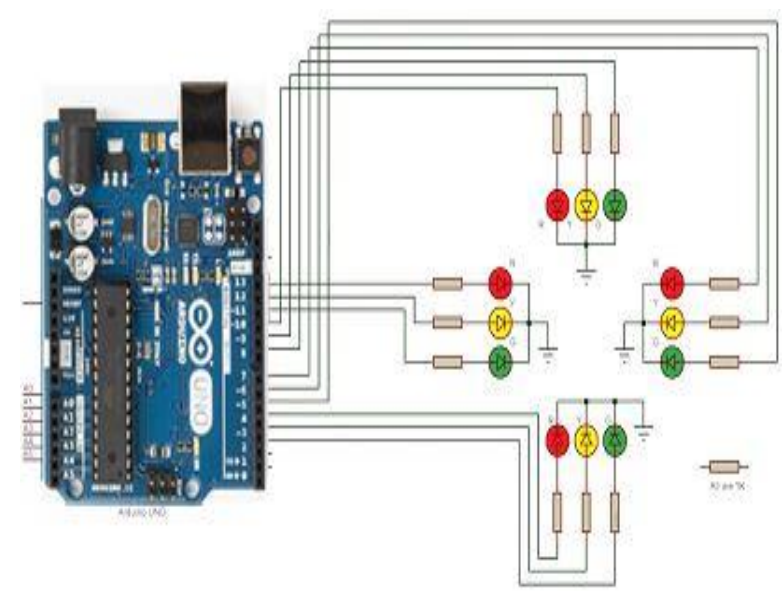

Fig 1: The circuit design.

\section{Circuit Components (particular part components)}

The components of the design circuit are:

1. Arduino UNO it is a microcontroller board built on ATmega328. It has a total of

- 14 digital input/output pins. 6 of those can be used as PWM outputs.
- 6 analog inputs

- A $16 \mathrm{MHz}$ of ceramic resonator

- a USB connection

- a power socket

- an ICSP header

- button to reset

It has complete functionality that is needed for the microcontroller. To get started, all that's needed to be done is to power it with an AC-to-DC adapter/ battery or alternatively it can also be connected to a computer through USB port. The latest Smart UNO development board version is Revision 3.

2. GSM (Global System for Mobile Communications, initially known as Group Spécial Mobile) is a standard developed by the European Telecommunications Standards Institute (ETSI). It is actually a set of protocols of mobile devices for $2 \mathrm{G}$ (second-generation) digital cellular networks which have widely replaced the $1 \mathrm{G}$ standard [6]. It originated in the December of 1991 in Finland[5].

Since 2014 it has been the global standard communication protocol for mobile networks. It is currently deployed in over 219 countries and enjoys 90\% market share. GSM is digitally optimized to give full duplex voice telephony that now includes data communication that was initially done by circuit switched transport and then by utilizing EDGE and GPRS (General Packet Radio Services) in packet data transport.

Subsequently, the 3GPP developed third-generation (3G) UMTS standards, followed by fourth-generation (4G) LTE Advanced standards, which do not form part of the ETSI GSM standard [6,7]

3. USB cable.

$4.1 \mathrm{~K} \Omega$ Resistor X 4.

$5.470 \Omega$ Resistor X 4.

6.Red LEDs X 4.

7. Yellow LEDs X 4.

8.Green LEDs X 4.

9.Connecting wires.

10.Prototyping board.

11.Power adapter. 


\section{The Second Circuit Design Using infrared Remote Control (IR):}

The second circuit design is by using IR control technology. It is a component of an electronic device used to operate the device wirelessly from a distance [9]. The receiver device is connected with the Arduino and the transmitter device is putted in ambulances or emergency vehicles. As shown in the form and when the driver of ambulances or emergency vehicles arrives at the traffic light, he can open the traffic signal by the remote which is located in the car. The IR controller is used in this experiment. It requires a clear line of distance to the receiver device for the signal, with a maximum of 30 feet $(9.14 \mathrm{~m})$ [8].

\section{Circuit Design:}

This circuit consists of the same first circuit design components but it replaces IR control instead of GSM. The circuit design is as shown in Figure 2 below.

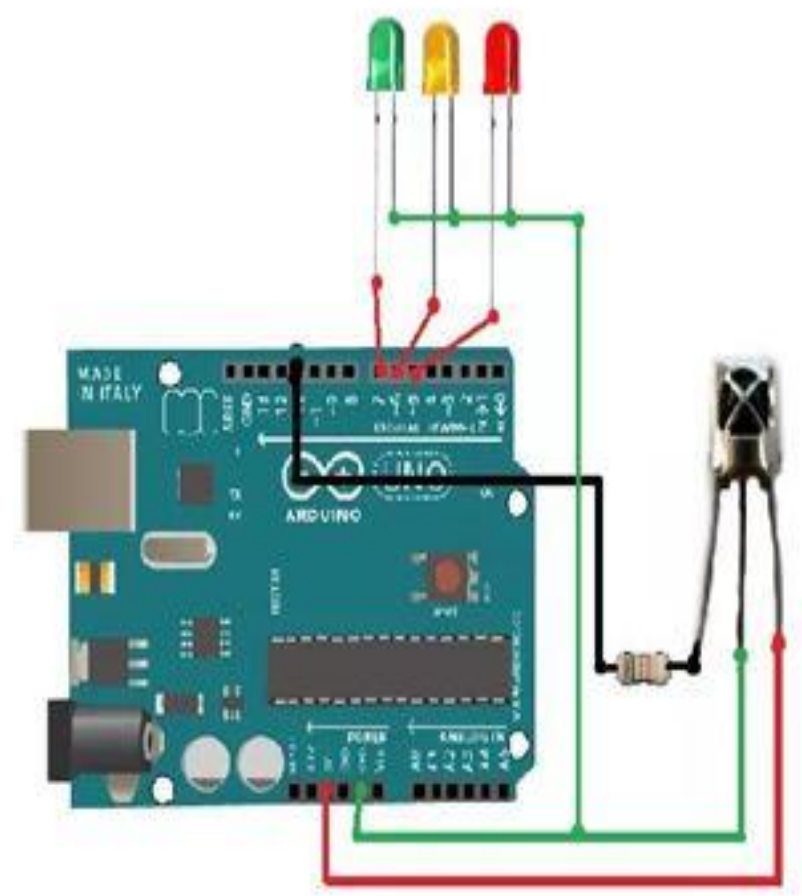

Figure 2: The Second Circuit Design Using (IR).

\section{The Proposed System}

In this study, a simple traffic light system for a 4 lanes intersection is implemented using Arduino UNO. The aim of the project is to implement a simple traffic light controller, where the traffic is controlled in a pre-defined timing system. Figure 3 below is the block diagram of the circuit design.

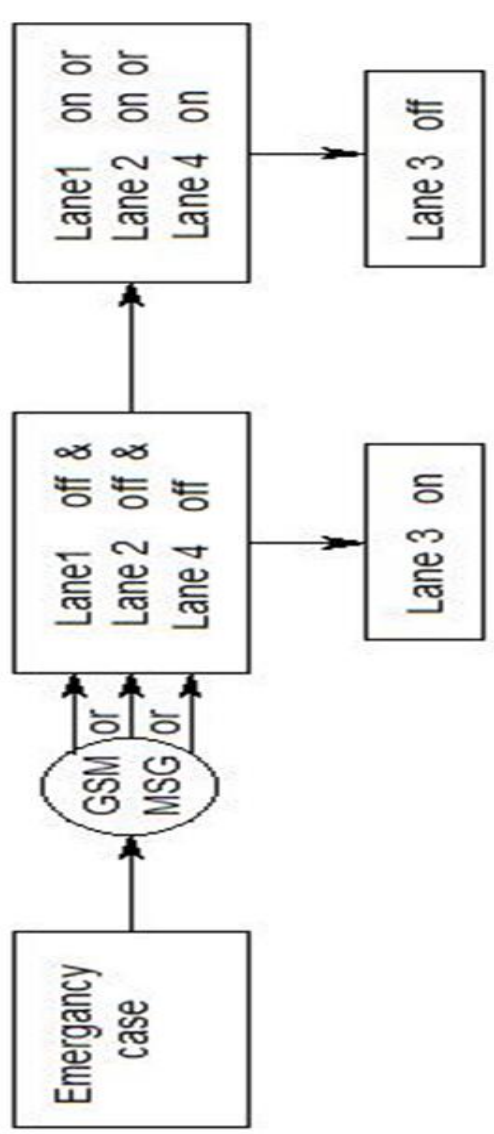

Figure 3: The Block Diagram of The Circuit Design. Suppose the first the Lane 1 gets its Green light turned. Hence, in all the other Lanes, their corresponding Red lights are turned on. The emergency case is in the third lane, then the vehicles driver (ambulance) can send message or signal by using GSM or IR to the traffic contrail and the lane 1 is turn off after the time is finished and red light turned, the lane 3 get its green must be open and another lanes gets Red lights turned, in this status the waiting time for emergence case becomes less than the waiting time of the normal case, As well we can reduce the traffic in the intersections by using this proposed. For example, if lane 2 has a lot of vehicles and lane 4 has few vehicles, in this case the waiting time causes crowded in the lane 2. To solve this problem, the time of lane 2 is increased and time of lane 4 is decreased. The working of the traffic light controller is as in Figure 4 below: 


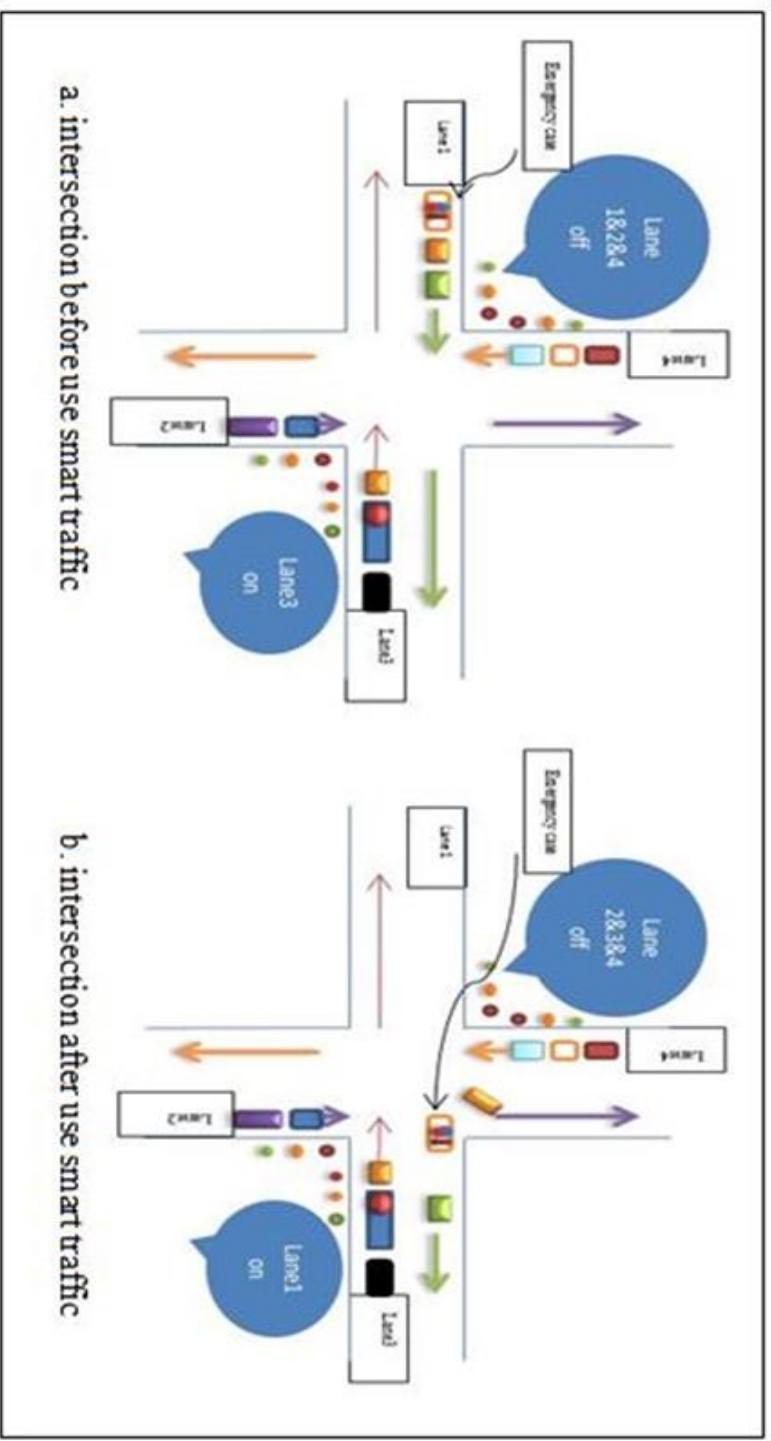

Figure 4: The Working of Traffic Light Controller

\section{COMPARISON}

\section{Comparison Between Our Two Circuits:}

The second circuit uses Remote control which works with infrared, the infrared works in $3 \mathrm{GHz}$ to $400 \mathrm{~Hz}$ [8], and the distance to which the radiation reaches is a few meters. While the first circuit (GSM), is not restricted by distance because it is use satellite technologies.

The GSM technology is not affected by obstacles such as walls and vehicles, whereas the remote control radiation is affected by the walls and vehicles. The radiation must be straight when attempting to open the traffic signal.

From the two experiments and the properties of each technique, we can approve that the use of the first circuit which it is use GSM is better than the second circuit which it is use the IR.

\section{Comparison between Our Circuits with Circuit of [1]:}

In [1], the smart traffic is designed to reduce the jam traffic in the road that had a lot of vehicles by using sensors. The sensors sense the vehicles number if there are many vehicles the system gives full time to that road. That mean, they reduced the jam traffic by changing the time.

In our work, we use GSM and IR for emergency case. Also, IR can be used by traffic cop when there is jam traffic in one of the road.

\section{ConClusion}

In this work, Arduino UNO was used to design smart traffic. Across road which consists of four lanes was designed. Each lane contained a traffic signal which consists of three lamps (red, yellow and green). The traffic light is controlled by Arduino. This work was designed for emergency cases, especially with ambulances by open the traffic signal in the lane which has the ambulance by using GSM system or IR. Where the ambulance send message or signal to the traffic light and open the lane. Our proposed system decreased the waiting time in the emergency cases. The GSM system was used in the first circuit and the IR system was used in the second circuit. After comparisons between the properties of these two circuits, we approved that the using of GSM is better than the using of IR. 


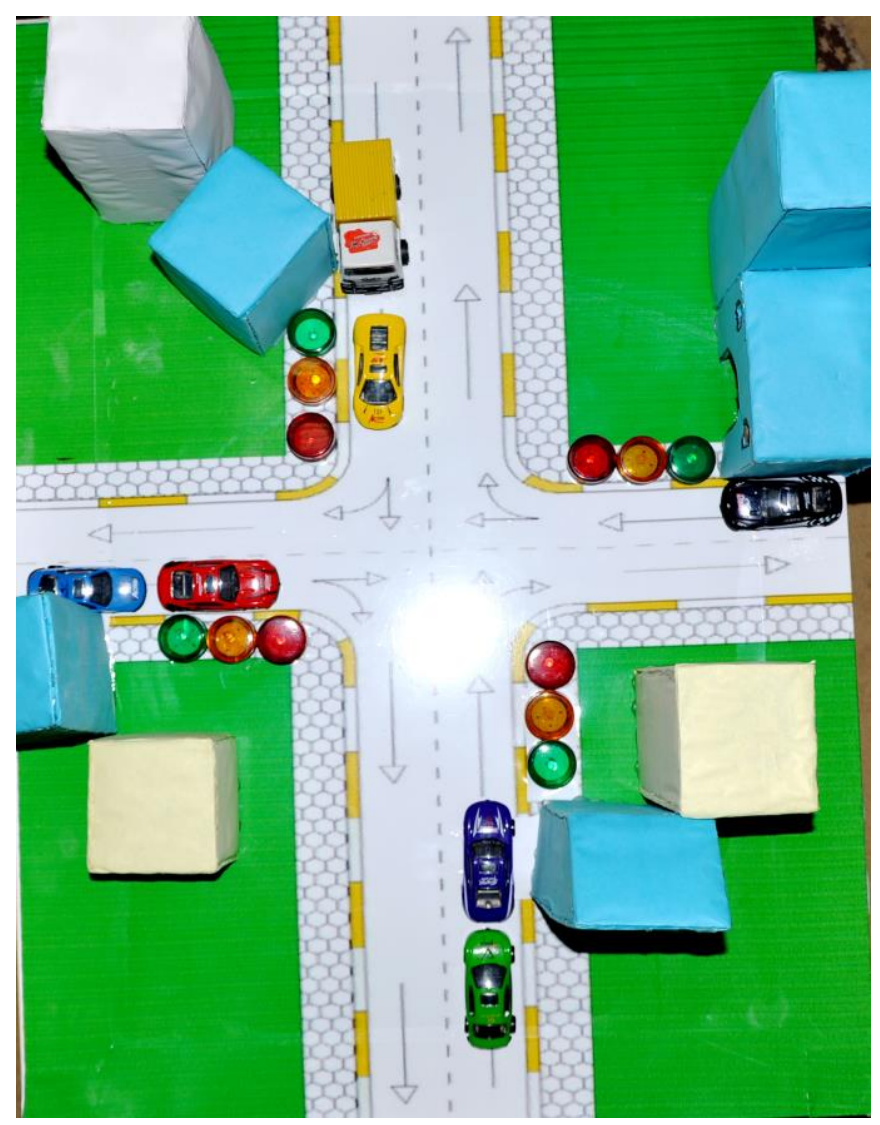

Figure 4: Smart Traffic Implementation

\section{References}

[1] A. AlBAgUl, H. HAMED, M. NAJI, A. ASSENI, A. ZARAGOUN, "Design and Fabrication of a Smart Traffic Light Control System," 3rd International conference Informatics and Computing Theory (AICT '12). Barcelona, Spain, 2012.

[2] Mohit Dev Srivastava1, Prerna2, Shubhendu Sachin3, Sumedha Sharma4, Utkarsh Tyagi "SMART TRAFFIC CONTROL SYSTEM USING PLC and SCADA" International Journal of Innovative Research in Science, Engineering and Technology, December 2012

[3] Dobre, C., Szekeres, A., Pop, F., \& Cristea, V.

[4] "Intelligent Traffic Lights to Reduce Vehicle Emissions". Proceedings 26th European Conference on Modeling and Simulation, Koblenz, Germany, 2012

[5] Abdul Kareem, E.I., Jantan, A., "An IntelligentTraffic Light Monitor System using an Adaptive Associative Memory", International Journal of Information Processing and Management, Vol.2. 2011

[6] Anton A. Huurdeman, The Worldwide History of Telecommunications, John Wiley \& Sons, 31 juli 2003, page 529.

[7] "GSM Global system for Mobile Communications". 4G Americas. Archived from the original on 8 February 2014. Retrieved 2014-03-22.

[8] "Maailman ensimmäinen GSM-puhelu" [World's first GSM call]. yle.fi. Yelisradio OY. 22 February 2008. Archived from the original on 5 May 2011. Retrieved 5 May 2011. Harri Holkeri made the first call on the Radiolinja (Elisa's subsidiary) network, at the opening ceremony in Helsinki on 07.01.1991.

[9] By Jim Lucas, "What is infrared?," live science, Missouri State University, March 26, 2015.

[10]"Remote control (desambiguación).," 18 September 201[Online].Available:http://en.wikipedia.org/wiki/Remote_control. [Accessed: 19 September 201].

[11]Hasan Omar Al-Sakran" Intelligent Traffic Information System Based on Integration of Internet of Things and Agent Technology" (IJACSA) International Journal of Advanced Computer Science and Applications, Vol. 6, No. 2, 2015

[12]Zhang Dahai, Jin Wenzhou and Wang Duanyi “ Analysis of the traffic problems and research on the traffic strategy in group urban development" college of traffic and communications, south china university of technology, guangzhou, guangdong 510640, china. 